\title{
A REMARK ON THE MEAN-FIELD DYNAMICS OF MANY-BODY BOSONIC SYSTEMS WITH RANDOM INTERACTIONS
}

\author{
WALID K. ABOU SALEM
}

\begin{abstract}
The mean-field limit for the dynamics of bosons with random interactions is rigorously studied. It is shown that, for interactions that are almost surely bounded, the many-body quantum evolution can be replaced in the mean-field limit by a single particle nonlinear evolution that is described by the Hartree equation. This is an Egorov-type theorem for many-body quantum systems with random interactions.
\end{abstract}

\section{INTRODUCTION}

This work is a modest contribution to the mathematical theory of the meanfield limit for bosons with random interactions. There has been substantial developments in the study of the mean-field dynamics of bosons with deterministic interactions. Early results were proven by Hepp in [1], see also [2]. A different approach based on the reduced density matrix was developed in [3] and was substantially extended to more general potentials and to the derivation of the Gross-Pitaevskii equation in [4, [5], 6], 7], 8]. Recently, a new approach was developed in 9], which gives convergence estimates in the mean-field limit that are uniform in Planck's constant $\hbar$, see also [10].

While the mean-field dynamics for bosons with deterministic interactions has attracted considerable interest, the question of the mean-field dynamics of bosons with random interactions has not been addressed, yet. Many-body bosonic systems with random interactions are relevant to concrete physical systems, such as inhomogeneous nonlinear optical media, or Bose-Einstein experiments where irregular fluctuations in currents inside conductors close to the condensate induce via Feshbach resonances inhomogeneous interactions between the bosons, see [1] for a description of the latter; also [12] and references therein. Here, we give a simple recepy for extending the deterministic mean-field analysis to the case of random interactions (and in the presence of a random potential).

1.1. The model. Consider the probability triple $(\Omega, \mathcal{F}, \mathbf{P})$, such that the probability space $\Omega$ has a generic point $\omega$ and is endowed with measure $\mu$. Define on this space the random field

$$
v(x, \omega) \quad: \quad \mathbb{R}^{3} \times \Omega \rightarrow \mathbb{R},
$$


such that $v$ is measurable in $x \in \mathbb{R}^{3}$ and $\omega \in \Omega$, and is almost surely in $L^{\infty}\left(\mathbb{R}^{3}\right)$, i.e. there exists $\Omega_{0} \subset \Omega$ such that $\mu\left(\Omega_{0}\right)=1$ and, for all $\omega \in \Omega_{0}, v(\cdot, \omega) \in L^{\infty}\left(\mathbb{R}^{3}\right)$. A concrete example of $v$ that satisfies the above conditions is $v(x, \omega)=v_{1}(x)+$ $v_{2}(x, \omega)$, such that $v_{1} \in L^{\infty}$ and $v_{2}$ is Gaussian with finite mean and variance. For a measurable and integrable function $f$ on $\Omega$, we define the expectation value of $f$ as

$$
\mathbb{E}(f):=\int f(\omega) \mu(d \omega) .
$$

We consider the $N$-body random Schrödinger operator

$$
H^{N} \equiv H_{\omega}^{N}:=-\sum_{i=1}^{N} \Delta_{i}+\frac{1}{N} \sum_{1 \leq i<j \leq N} v\left(x_{i}-x_{j}, \omega\right)
$$

where $\Delta=\sum_{j=1}^{3} \frac{\partial^{2}}{\partial x_{j}^{2}}$ is the 3 -dimensional Laplacian and $\omega \in \Omega$. Here, we work in units where Planck's constant $\hbar=1$ and the mass of each particle is $m=\frac{1}{2}$. We note that the analysis below is uniform in $\hbar$. The Hamiltonian $H^{N}$ acts on the Hilbert space $\mathcal{H}^{N}:=L_{S}^{2}\left(\mathbb{R}^{3 N}\right)$, the symmetrization of $L^{2}\left(\mathbb{R}^{3 N}\right)$, which is the space of pure states for a system of $N$ nonrelativistic bosons.

The quantum dynamics of the $N$-body system is described by the Schrödinger equation

$$
i \partial_{t} \Psi^{N}(t)=H^{N} \Psi^{N}(t)
$$

with an initial condition $\Psi^{N}(t=0)=\Psi^{N, 0} \in L_{S}^{2}\left(\mathbb{R}^{3 N}\right)$.

Together with the dynamics defined above, the $N$-body system is described by a kinematical algebra of "observables". For $p \leq N$, a $p$-particle observable is described by an operator $a^{(p)} \in \mathcal{B}\left(\mathcal{H}^{(p)}\right)$, where $\mathcal{B}\left(\mathcal{H}^{(p)}\right)$ is the algebra of bounded operators on $\mathcal{H}^{(p)}=L_{S}^{2}\left(\mathbb{R}^{3 p}\right)$. By the nuclear theorem, one can associate with $a^{(p)}$ a tempered distribution kernel in $\mathcal{S}^{\prime}\left(\mathbb{R}^{3 p} \times \mathbb{R}^{3 p}\right), \alpha^{(p)}\left(x_{1}, \ldots, x_{p} ; y_{1}, \ldots, y_{p}\right):=$ $\alpha^{(p)}\left(X_{p} ; Y_{p}\right)$, such that

$$
\left(a^{(p)} \varphi^{(p)}\right)\left(X_{p}\right)=\int_{\mathbb{R}^{3 p}} \alpha^{(p)}\left(X_{p} ; Y_{p}\right) \varphi^{(p)}\left(Y_{p}\right) d Y_{p}
$$

where $\varphi^{(p)}\left(Y_{p}\right) \in L_{S}^{2}\left(\mathbb{R}^{3 p}\right)$. We associate to $a^{(p)}$ an operator $A^{N}\left(a^{(p)}\right)$ acting on $\mathcal{H}^{(N)}$ that is given by

$$
\left(A^{N}\left(a^{(p)}\right) \Psi\right)\left(x_{1}, \cdots, x_{N}\right)=\frac{N !}{N^{p}(N-p) !}\left(P_{S} a^{(p)} \otimes I^{(N-p)} P_{S} \Psi\right)\left(x_{1}, \ldots, x_{N}\right),
$$

where $\Psi\left(x_{1}, \ldots, x_{N}\right) \in L_{S}^{2}\left(\mathbb{R}^{3 N}\right)$ and $P_{S}$ is the projection onto the symmetric subspace $L_{S}^{2}\left(\mathbb{R}^{3 N}\right)$ of $L^{2}\left(\mathbb{R}^{3 N}\right)$. It follows from (3) and (4) that the map

$$
A^{N}: \mathcal{B}\left(\mathcal{H}^{(p)}\right) \rightarrow \mathcal{B}\left(\mathcal{H}^{(N)}\right), \quad 1 \leq p \leq N
$$


is linear, such that

$$
\begin{aligned}
& \left\|A^{N}\left(a^{(p)}\right)\right\|_{\mathcal{B}\left(\mathcal{H}^{(N)}\right)} \leq\left\|a^{(p)}\right\|_{\mathcal{B}\left(\mathcal{H}^{(p)}\right)}, \\
& A^{N}\left(a^{(p)}\right)^{*}=A^{N}\left(a^{(p) *}\right) .
\end{aligned}
$$

In the Heisenberg picture, the evolution of $A^{(N)} \in \mathcal{B}\left(\mathcal{H}^{(N)}\right)$ is given by

$$
\alpha_{t}^{N}\left(A^{(N)}\right):=e^{i H^{N} t} A^{(N)} e^{-i H^{N} t}, \quad t \in \mathbb{R} .
$$

Since $v$ is almost surely bounded, $H^{N}$ is almost surely self-adjoint on the symmetrized Sobolev space $H_{S}^{2}\left(\mathbb{R}^{3 N}\right)$, and hence the propagator $e^{-i H^{N} t}, \quad t \in \mathbb{R}$, is almost surely unitary. Moreover, it follows from the fact that the pointwise limit of measurable functions is itself measurable, [13], and the Trotter product formula, 14, that

$$
\left\langle\otimes_{j=1}^{N} \psi_{j}\left(x_{j}\right), \alpha_{t}^{N}\left(A^{(N)}\right) \otimes_{j=1}^{N} \psi_{j}\left(x_{j}\right)\right\rangle, \quad A^{(N)} \in \mathcal{B}\left(\mathcal{H}^{(N)}\right), \quad \psi_{j} \in L^{2}\left(\mathbb{R}^{3}\right)
$$

is $\omega$-measurable.

We now introduce the classical evolution. The Hartree equation is given by

$$
i \partial_{t} \psi_{t}=-\Delta \psi_{t}+\left(v \star\left|\psi_{t}\right|^{2}\right) \psi_{t}
$$

with the initial condition $\psi_{t=0}=\phi \in L^{2}\left(\mathbb{R}^{3}\right)$. It follows from Duhamel's formula for $\psi_{t}$ and the fact that $v \in L^{\infty}$ almost surely, that global solutions of (17) in $L^{2}$ exist almost surely, such that $\left\|\psi_{t}\right\|_{L^{2}}=\|\phi\|_{L^{2}}$ with probability 1 , for all $t \in \mathbb{R}$, (see for example [15] for the case when $v \in L^{\infty}$ ). It also follows from Duhamel's formula that the random variable

$$
\left\langle\otimes_{i=1}^{p} \psi_{t}, A^{(p)} \otimes_{i=1}^{p} \psi_{t}\right\rangle, \quad A^{(p)} \in \mathcal{B}\left(\mathcal{H}^{(p)}\right)
$$

is $\omega$-measurable.

1.2. Statement of the main result. We are in a position to state the main result.

Theorem 1. Given $a^{(p)}, A^{N}\left(a^{(p)}\right)$ and $\alpha_{t}^{N}$ as above, suppose that the initial state of the $N$-body system is a normalized coherent (product) state $\Psi^{N, 0}\left(x_{1}, \cdots, x_{N}\right)=$ $\otimes_{i=1}^{N} \phi\left(x_{i}\right), \quad \phi \in L^{2}\left(\mathbb{R}^{3}\right)$. Then, for fixed $t \geq 0$,

$$
\lim _{N \rightarrow \infty} \mathbb{E}\left(\left\langle\Psi^{N, 0}, \alpha_{t}^{N}\left(A^{N}\left(a^{(p)}\right)\right) \Psi^{N, 0}\right\rangle\right)=\mathbb{E}\left(\left\langle\otimes_{i=1}^{p} \psi_{t}, a^{(p)} \otimes_{i=1}^{p} \psi_{t}\right\rangle\right),
$$

where $\psi_{t}$ satisfies the Hartree equation (7) with initial condition $\psi_{t=0}=\phi$.

We note that the analysis below can be easily extended to study the mean-field dynamics of bosons in a random external potential that is almost surely smooth, polynomially bounded and positive, and to investigate the semi-classical limit of the dynamics under additional assumptions on the decay of the interaction, as in [9]. Furthermore, the analysis below can be applied "in toto" to extend the results of [10] and [16] to the case of random interactions. 


\section{Proof of Theorem 1}

The proof of Theorem 1 follows effectively from an application of the dominated convergence theorem, see [13], and Theorem 1.1 in [9]. In what follows, we drop the explicit dependence on the time $t$ in the notation, since we fix it.

Proof. We introduce the random variables

$$
X_{N}^{(p)}:=\left\langle\Psi^{N, 0}, \alpha_{t}^{N}\left(A^{N}\left(a^{(p)}\right)\right) \Psi^{N, 0}\right\rangle
$$

and

$$
X^{(p)}:=\left\langle\otimes_{i=1}^{p} \psi_{t}, a^{(p)} \otimes_{i=1}^{p} \psi_{t}\right\rangle
$$

The claim of the theorem is equivalent to the statement

$$
\lim _{N \rightarrow \infty} \mathbb{E}\left(X_{N}^{(p)}\right)=\mathbb{E}\left(X^{(p)}\right) .
$$

We divide the proof of (8) into several steps. Step 1. Uniform integrability. We want to show that

$$
\lim _{\beta \rightarrow \infty} \mathbb{E}\left(\left|X_{N}^{(p)}\right| \mathbf{1}_{\left|X_{N}^{(p)}\right| \geq \beta}\right)=0
$$

uniformly in $N \in \mathbb{N}$.

We have from (5) and the fact that the quantum time-evolution is almost surely unitary, that

$$
\left|X_{N}^{(p)}\right| \leq\left\|a^{(p)}\right\|_{\mathcal{B}\left(\mathcal{H}^{(p)}\right)}<2\left\|a^{(p)}\right\|_{\mathcal{B}\left(\mathcal{H}^{(p)}\right)}<\infty, \text { almost surely }
$$

uniformly in $N \in \mathbb{N}$. For $\beta>0$, it follows from (10) that

$$
\left|X_{N}^{(p)}\right| \mathbf{1}_{\left|X_{N}^{(p)}\right| \geq \beta} \leq\left|X_{N}^{(p)}\right|<2\left\|a^{(p)}\right\|_{\mathcal{B}\left(\mathcal{H}^{(p)}\right)}<\infty \text {, almost surely, }
$$

uniformly in $N \in \mathbb{N}$. The dominated convergence theorem together with (11) give (9).

Step 2. Mean-field limit with probability 1. It follows from the fact that the particle interaction $v \in L^{\infty}$ almost surely and Theorem 1.1 in [9] that, for fixed $t>0$

$$
X_{N}^{(p)} \stackrel{N \rightarrow \infty}{\rightarrow} X^{(p)} \text { almost surely. }
$$

Step 3. It follows from Fatou's lemma, [13], and (10), that

$$
\mathbb{E}\left(\left|X^{(p)}\right|\right) \leq \liminf _{N} \mathbb{E}\left(\left|X_{N}^{(p)}\right|\right) \leq \limsup _{N} \mathbb{E}\left(\left|X_{N}^{(p)}\right|\right)<2\left\|a^{(p)}\right\|_{\mathcal{B}\left(\mathcal{H}^{(p)}\right)}<\infty,
$$

uniformly in $N \in \mathbb{N}$. We also have that

$$
\left|X^{(p)}\right| \mathbf{1}_{\left|X^{(p)}\right| \geq \beta} \leq\left|X^{(p)}\right|
$$

which together with (13) and the dominated convergence theorem, imply that

$$
\lim _{\beta \rightarrow \infty} \mathbb{E}\left(\left|X^{(p)}\right| \mathbf{1}_{\left|X^{(p)}\right| \geq \beta}\right)=0 .
$$


Step 4. Convergence as $N \rightarrow \infty$. We introduce the random variable

$$
Y_{N}^{(p)}:=\left|X^{(p)}-X_{N}^{(p)}\right| \text {. }
$$

Note that it suffices to show that $\mathbb{E}\left(Y_{N}^{(p)}\right) \rightarrow 0$ as $N \rightarrow \infty$, from which (8) follows by the triangular inequality.

It follows from (12), Step 2, that

$$
Y_{N}^{(p)} \stackrel{N \rightarrow \infty}{\rightarrow} 0 \text { almost } \text { surely. }
$$

We decompose $Y_{N}^{(p)}$ into two parts,

$$
Y_{N}^{(p)}=Y_{N}^{(p),<\beta}+Y_{N}^{(p), \geq \beta}
$$

where $Y_{N}^{(p),<\beta}:=Y_{N}^{(p)} \mathbf{1}_{\left|Y_{N}^{(p)}\right|<\beta}$ and $Y_{N}^{(p), \geq \beta}:=Y_{N}^{(p)} \mathbf{1}_{\left|Y_{N}^{(p)}\right| \geq \beta}$, for $\beta>0$.

Since $Y_{N}^{(p),<\beta}<\beta$, (15) together with the dominated convergence theorem imply that

$$
\lim _{N \rightarrow \infty} \mathbb{E}\left(Y_{N}^{(p),<\beta}\right)=0
$$

Furthermore, since

$$
Y_{N}^{(p), \geq \beta} \leq 2\left|X^{(p)}\right| \mathbf{1}_{\left|X^{(p)}\right| \geq \beta / 2}+2\left|X_{N}^{(p)}\right| \mathbf{1}_{\left|X_{N}^{(p)}\right| \geq \beta / 2},
$$

it follows from (9) and (14) that

$$
\lim _{\beta \rightarrow \infty} \mathbb{E}\left(Y_{N}^{(p), \geq \beta}\right)=0
$$

uniformly in $N \in \mathbb{N}$.

Given $\epsilon>0$, (17) implies that there exists a finite $\beta_{0}>0$ such that

$$
\sup _{N} \mathbb{E}\left(Y_{N}^{(p), \geq \beta_{0}}\right)<\epsilon / 2
$$

Moreover, (16) implies that there exists a positive integer $N_{0}$ such that, for all $N \geq N_{0}$

$$
\mathbb{E}\left(Y_{N}^{(p),<\beta_{0}}\right)<\epsilon / 2
$$

It follows that

$$
\mathbb{E}\left(Y_{N}^{(p)}\right)=\mathbb{E}\left(Y_{N}^{(p),<\beta_{0}}\right)+\mathbb{E}\left(Y_{N}^{(p), \geq \beta_{0}}\right)<\epsilon
$$

for $N \geq N_{0}$. Therefore, $\mathbb{E}\left(Y_{N}^{(p)}\right) \stackrel{N \rightarrow \infty}{\rightarrow} 0$.

By the triangular inequality,

$$
\left|\mathbb{E}\left(X^{(p)}\right)-\mathbb{E}\left(X_{N}^{(p)}\right)\right| \leq \mathbb{E}\left(Y_{N}^{(p)}\right) \stackrel{N \rightarrow \infty}{\rightarrow} 0,
$$

which gives the claim of the theorem. 


\section{REFERENCES}

[1] K. Hepp. The classical limit for quantum mechanical correlation functions. Comm. Math. Phys. 35: 265-267, 1974.

[2] J. Ginibre, G. Velo. On a class of nonlinear Schrödinger equations with nonlocal interaction. Math. Z., 170: 109 - 136, 1980.

[3] H. Spohn. Kinetic equations from Hamiltonian dynamics: Markovian limits. Rev. Mod. Phys. 53: 569 - 615, 1980.

[4] L. Erdos and H.-T. Yau. Derivation of the nonlinear Schrödinger equation from a many body Coulomb system. Adv. Theor. Math. Phys., 5: 1169, 2001.

[5] C. Bardos, F. Golse, N. Mauser. Weak coupling limit of the $N$-particle Schrödinger equation. Methods Appl.Anal. 2: 275 - 293, 2000.

[6] C. Bardos, L. Erdös, F. Golse, N. Mauser and H-T Yau. Derivation of the SchrödingerPoisson equation from the quantum N-body problem. C.R. Acad. Sci. Paris, 334: 515 $520,2002$.

[7] L. Erdös, B. Schlein and H.-T. Yau. Derivation of cubic nonlinear Schrödinger equation from quantum dynamics of many-body systems. Invent. Math., 167: 515, 2007.

[8] L. Erdös, B. Schlein and H.-T. Yau. Derivation of the Gross-Pitaevskii equation for the dynamics of Bose-Einstein condensates. Preprint 2006.

[9] J. Fröhlich, S. Graffi and S. Schwarz. Mean-field and classical limit of many-body Schrödinger dynamics for bosons. Commun. Math. Phys., 271: 681, 2007.

[10] J. Fröhlich, A. Knowles and A. Pizzo. Atomism and quantization. J. Phys. A, 40:3033, 2007.

[11] S. Wildermoth et al.. Bose-Einstein condensates: microscopic magnetic field imaging. $\mathrm{Na}$ ture, 435: 440, 2005.

[12] F. Kh. Abdullaev and J. Garnier. Propagation of matter wave solitons in periodic and random nonlinear potentials. Phys. Rev. A, 72: 061605R, 2005.

[13] P. Billingsley. Probability and Measure, $3^{\text {rd }}$ edition. John Wiley and Sons, New York, 1995.

[14] M. Reed and B. Simon. Methods of Modern Mathematical Physics II: Fourier Analysis, Self-Adjointness. Academic Press, San Diego, 1975.

[15] T. Cazenave. An Introduction to Nonlinear Schrödinger Equations. Textos de Métodos Matemáticos 26. Instituto de Matemática, Rio de Janeiro, 1996.

[16] J. Fröhlich, A. Knowles and E. Lenzmann. Semi-classical dynamics in quantum spin systems. Preprint 2007.

Department of Mathematics, University of Toronto, Toronto, Ontario, Canada M5S 2E4, E-MAIL: WALID@MATH.UTORONTO.CA 